\title{
Improving Quality in Healthcare: Translating Evidence into Practice
}

\author{
Authors
}

Paula Elouafkaoui - DHSRU, University of Dundee/TRiaDS, NHS Education for Scotland

Heather Cassie - DHSRU, University of Dundee

\section{Background}

A consistent finding in health services research is that the translation of research findings into practice is unpredictable and can be a slow and haphazard process. ${ }^{1}$ It is also well documented that the translation of guidelines into clinical practice requires more than the publication of evidence-based clinical guidelines alone. ${ }^{2-5}$ Established in 2008, the Translation Research in a Dental Setting (TRiaDS) Programme ${ }^{5}$ is a multi-disciplinary collaboration that has been formed to develop a programme of knowledge translation research embedded within the Scottish Dental Clinical Effectiveness Programme (SDCEP) guidance development process. The TRiaDS Research Methodology Group (RMG) has public, academic, policy, service and professional members, including external national and international leaders in the area of knowledge translation science.

TRiaDS aims to develop and evaluate implementation strategies to support the translation of knowledge into dental practice.
It offers the potential to create a research laboratory for the provision and exchange of evidence-based information between the TRiaDS RMG, dental healthcare professionals, educators and policy makers on how best to translate service and educational initiatives into routine clinical practice.

\section{TRiaDS Objectives}

1. To investigate the need for active knowledge translation strategies for SDCEP guidance using an appropriate mix of qualitative and quantitative methods

2. To develop theoretically informed knowledge translation strategies to support implementation of SDCEP guidance

3. To evaluate the effectiveness, cost effectiveness and sustainability of a range of knowledge translation interventions using experimental designs

4. To synthesise knowledge gained from multiple and sequential knowledge translation evaluations using a theoretical framework
5. Through the conduct of the programme to inform practitioners, education providers, policy makers and patients on how to effectively and efficiently translate national recommendations into routine clinical activities.

\section{TRiaDS Methodology}

For each guidance document a diagnostic analysis begins at the start of guidance development. Information is gathered about current practice; key recommendations and associated behaviours are identified and prioritised; and stakeholder questionnaires and interviews are used to identify potential barriers and facilitators. Where possible, routinely collected data are used to measure compliance with the guidance and to inform decisions about whether a knowledge translation intervention is required.

\section{Conference}

Co-organised by NHS Education for Scotland (NES) and the Health Services Research Unit

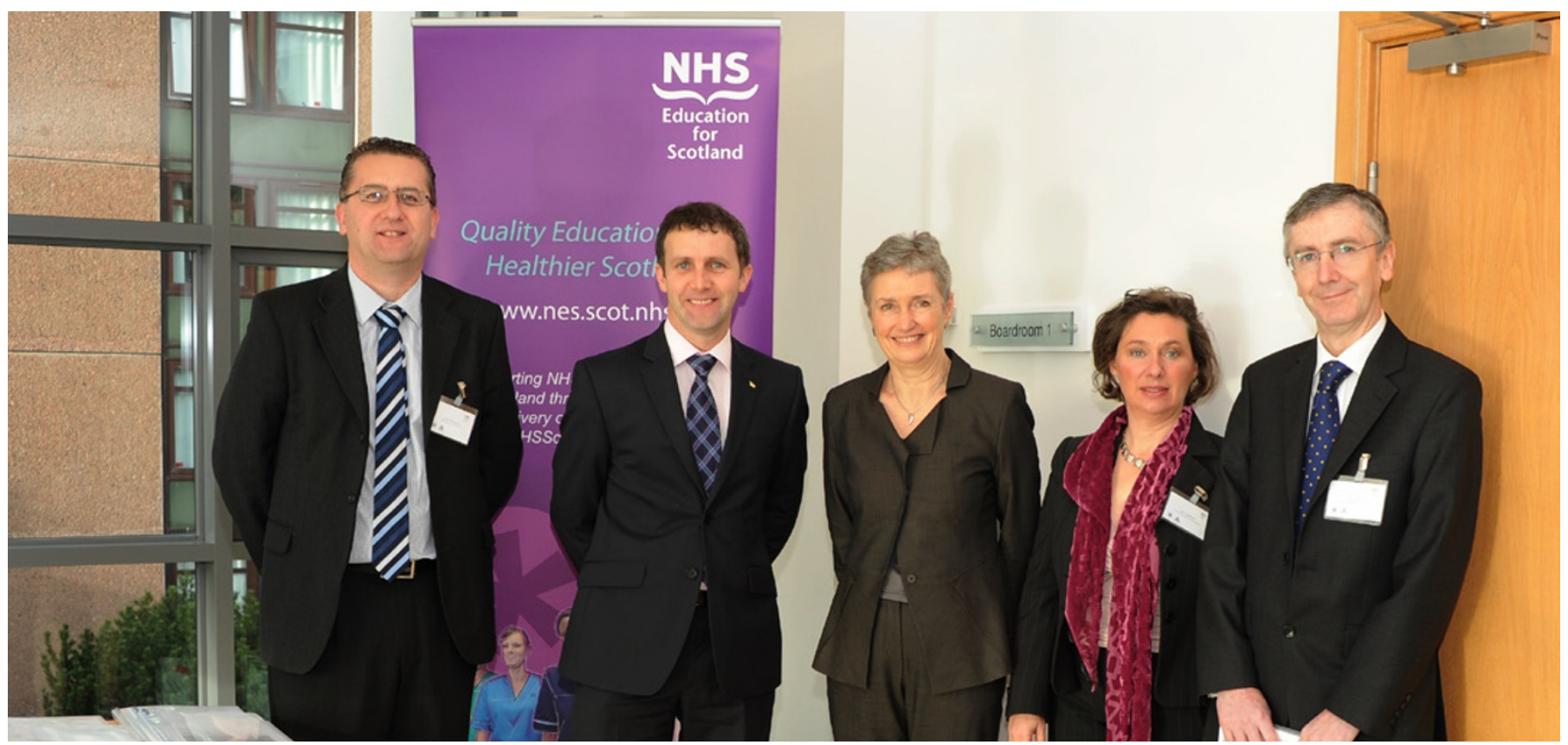

Fig 1 From left to right, Prof. Craig Ramsay, Michael Matheson, Minister for Public Health and Sport, Dr Lindsay Burley, Prof. Jan Clarkson, Dr. David Felix 
(HSRU) at the University of Aberdeen, TRiaDS hosted their first international conference on the $7^{\text {th }}$ November 2012. The event, held at the John McIntyre Conference Centre in Edinburgh, saw over 100 practitioners, academics, students and decision-makers engaged in the development and translation of evidence-based knowledge gather to hear experts in the field who shared their knowledge and insights through interactive presentations and discussion sessions.

Dr Lindsay Burley, Chair of NES, opened proceedings for the day and introduced the first speaker, Professor Jeremy Grimshaw, Senior Scientist of the Clinical Epidemiology Programme at Ottawa Hospital Research Unit. Professor Grimshaw addressed the challenges that surround knowledge translation, focussing on how knowledge translation strategies can improve care, how these strategies themselves can be improved upon and how to embed knowledge translation into healthcare systems.

The second address was by Michel Wensing, Professor of Implementation Science at Rabound University in the Netherlands. He discussed the impact of implementation science on healthcare practice and policy, illustrating with examples of ongoing primary care research in the Netherlands and Europe.

The keynote speaker, Michael Matheson, Minister for Public Health and Sport, brought the first session to a close. Mr Matheson highlighted initiatives such as SDCEP, and explained how the work of TRiaDS is embedded within the SDCEP guidance development process. The importance of translating research findings into clinical practice was also emphasised. The minister also touched upon the important work of the Childsmile programme and how this has helped to bring about improvements in the oral health of children in Scotland. The NESdeveloped Knowledge Network, Scotland's national online gateway to knowledge for health and social care, also featured.

A series of parallel sessions followed with presenters from a range of organisations, including; NICE, the UK Cochrane Centre, Healthcare Improvement Scotland, University College London and the Universities of Leicester, Leeds and Aberdeen. These sessions focussed on knowledge synthesis, knowledge translation and examples of knowledge translation research.

Martin Eccles, Professor of Clinical Effectiveness at the University of Newcastle,

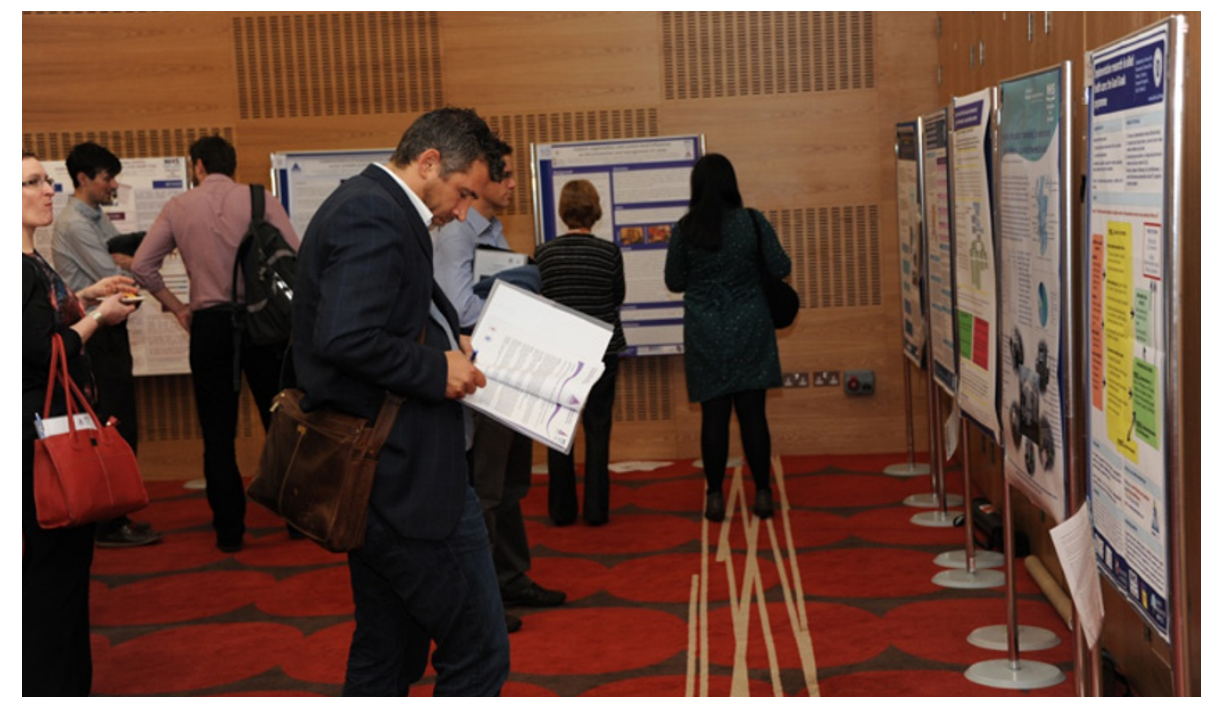

Fig 2. Poster viewing at the conference

gave the final presentation of the day. He identified and reflected on the potential political and scientific challenges that knowledge translation research may have to address in the future.

Audience participation was encouraged throughout the event, with delegates voting for the winner in the Scottish Dental Practice Based Research Network (SDPBRN) sponsored poster competition. Posters covered a range of studies in the areas of knowledge translation research and evaluation and were very well received by the audience. The majority of those who completed the online evaluation form rated them as either very good or excellent. Michal Meszka, presenting author of the winning poster, 'An Audit to Address the Problem of Inappropriate New Patient Referrals for Orthodontics in the Northern Isles' was presented with a book token on behalf of SDPBRN. The day ended with a lively question and answer session, chaired by Professor Jan Clarkson, where pre-submitted questions from conference delegates where put to the keynote speakers.

The event was branded a huge success, with $92 \%$ of those who provided feedback rating the event as either very good or excellent. Many felt the conference provided good networking opportunities, and most indicated they would probably attend another conference in this field. Speaking after the conference Professor Jan Clarkson, Director of SDCEP and co-chair of the TRiaDS RMG group, was delighted with the success of the event. She commented: "Today was a fantastic opportunity to bring together a range of practitioners and international knowledge translation researchers to provide a forum to share experiences and expertise.
We plan to build on the success of today with future events that will continue to motivate those undertaking research in this area.'

\section{Current Projects}

TRiaDS have a number of ongoing projects at various stages of the SDCEP guidance development process. For example, dental hygienists have recently completed a survey to help inform new guidance on the prevention and treatment of periodontal diseases in primary care. A mixed methods study is being conducted to explore the patient, organisation and system level barriers and facilitators to the prevention and management of caries in general dental practice. In recent weeks dentists and doctors have been asked to complete surveys in relation to the management of patients prescribed bisphosphonates, and a national randomised controlled trial to evaluate the effectiveness of audit and feedback strategies on reducing antibiotic prescribing in dentistry (RAPiD) is currently underway.

For more information on TRiaDS or any of the above studies, please contact TRiaDS@ nes.scot.nhs.uk.

1. Seddon ME, Marshall MN, Campbell SM, Roland MO. Systemic review of studies of quality of clinical care in general practice in the UK, Australia and New Zealand. Qual Health Care 2001; 10: 152-158.

2. Schuster MA, McGlynn EA, Brook RH. How good is the quality of health care in the United States? Milbank Q 1998; 76: 517-563.

3. Grol R. Successes and failures in the implementation of evidence-based guidelines for clinical practice. Med Care 2001; 39: II46-II54.

4. McGlynn EA, Asch SM, Adams ], et al. The quality of health care delivered to adults in the United States. N Engl J Med 2003; 348: 2635-2645.

5. Clarkson JE, Ramsay CR, Eccles MP, et al. The translation research in a dental setting (TRiaDS) programme protocol. Implementation Sci 2010; 5: 57.

Evidence-Based Dentistry (2013) 14, 3-4 doi:10.1038/sj.ebd.6400908 